\title{
Management of Left Ventricular Aneurysm: A Study from Iraq
}

\author{
Abdulsalam Y. Taha ${ }^{1 *}$, Bassam A. Mahmoud ${ }^{2}$ \\ ${ }^{1}$ Department of Thoracic and Cardiovascular Surgery, School of Medicine and Sulaimania Teaching Hospital, Sulaimania, Iraq; \\ ${ }^{2}$ Department of Cardiac Surgery, Ibn-Albitar Center for Cardiac Surgery (IBCCS), Baghdad, Iraq. \\ Email: salamyt_1963@hotmail.com
}

Received November $28^{\text {th }}, 2013$; revised December $25^{\text {th }}, 2013$; accepted January $20^{\text {th }}, 2014$

Copyright (C) 2014 Abdulsalam Y. Taha, Bassam A. Mahmoud. This is an open access article distributed under the Creative Commons Attribution License, which permits unrestricted use, distribution, and reproduction in any medium, provided the original work is properly cited. In accordance of the Creative Commons Attribution License all Copyrights (c) 2014 are reserved for SCIRP and the owner of the intellectual property Abdulsalam Y. Taha, Bassam A. Mahmoud. All Copyright (C) 2014 are guarded by law and by SCIRP as a guardian.

\section{ABSTRACT}

Background: The most appropriate surgical approach for post-myocardial infarction left ventricular aneurysm (LVA) is controversial. This study aims to display the results of surgical treatment of LVA in a major Iraqi cardiac surgical center. Methods: The surgical management of LVAs over the period 2001 to 2011 was retrospectively reviewed. The presenting signs and symptoms, results of investigations, operative findings, and outcomes of patients were determined. Results: Twenty-seven true LVAs associated with 4 ventricular septal defects (VSDs) were treated surgically. During the same period, 1136 coronary artery bypass graft (CABG) operations were done, thus LVA represented $2.4 \%$. Males constituted the majority $(\mathbf{7 4 . 1 \% )}$. The mean age was 54.6 years old.

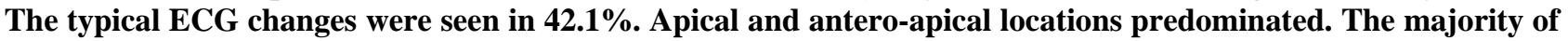
patients (84.2\%) had subnormal values of ejection fraction (EF). Most patients had multi-vessel coronary artery disease (CAD). The most frequent was the left anterior descending artery (LAD). All patients had CABG except 3. Linear repair and Dor technique were used equally. The commonest postoperative complication was bleeding (38.4\%). The overall hospital mortality was $\mathbf{1 8 . 5 \%}$. Conclusion: Concomitant CABG improves early postoperative course and must be added when significant lesions in coronary arteries particularly the LAD are present.

\section{KEYWORDS}

\section{Left Ventricular Aneurysm; Post-Ischemic VSD; Linear Repair and Dor Technique}

\section{Introduction}

LVAs have long been described at autopsy, but LVA was not recognized to be a consequence of coronary artery disease until 1881 [1]. The angiographic diagnosis of LVA was first made in 1951 [1]. The surgical treatment of ventricular aneurysm was introduced in 1944, when Claude S. Beck reinforced the wall of LVA with fascia lata aponeurosis in order to reduce excessive dilatation and avoid LVArupture. In 1955, Likoff and Bailey performed closed ventriculotomy by placing a large vascular clamp on the beating heart tangentially across the base of LVA followed by resection and suture [2]. In 1958, Cooley and colleaues performed the first postinfarction

*Corresponding author. aneurysm resection and linear repair of left ventriculotomy with the use of cardiopulmonary bypass. The technique offered by Levinsky and colleagues in 1979 supposed the performance of left ventricular reconstruction with a woven Dacron patch after resection of anterior postinfarction aneurysm. In 1985, Jatene and Dor independetly presented a fundamentally new, anatomic left ventricular reconstruction method with endoventricular circular reduction and stiching patch in the formed ventriculotomy orifice [3].

The aim of this paper was to study the management of true LVA following MI in a major Iraqi cardiac surgical centre noting the methods of diagnosis and surgical treatment options and the outcome in view of the relevant literature. 


\section{Materials and Methods}

Twenty-seven patients (20 males and 7 females) with LVA who were admitted to Ibn-Albitar Centre for Cardiac Surgery (IBCCS) over the period from May $1^{\text {st }}$, 2001 to December $31^{\text {st }}$, 2011 were retrospectively studied. Patients' informed consents and approval of the Hospital Ethics Committee were obtained. The case sheets of these patients were obtained. Information like age, sex, place of residency, presenting symptoms and signs, past medical history particularly ischemic heart disease (IHD) were looked for. The diagnostic work-up was reviewed looking for specific investigations like electrocardiography (ECG), chest radiography (CXR), echocardiography, cardiac catheterization and coronary angiography. All patients in this study were initially seen and thoroughly investigated by cardiologists. Thereafter, surgical candidates were referred for surgery.

All patients had repair of aneurysm (mostly linear repair or Dor procedure) together with myocardial revascularization (CABG) except 3 patients who had repair of their aneurysm alone. Four patients with an associated VSD had closure of this defect as well. The operative notes and the perfusionists' notes were all reviewed to get an idea of the conduct of the operation. Intra-aortic balloon pump (IABP) was used selectively (either before induction of anesthesia or at the end of operation).

Operative procedure: The aneurysmal wall was incised and thrombi removed if present. Repair was done either by the linear method for small aneurysms or Dor procedure for big ones. In the linear method, after excising the aneurysm, the edges of viable myocardium were sutured together by interrupted pledgeted sutures over Teflon felts. While in Dor technique, a purse string (2 zero) Polypropylene suture was used to narrow the defect. The remaining ventriculotomy was closed by a Dacron patch sutured interruptedly by pledgeted Polypropylene sutures over Teflon strips.

The postoperative morbidity and mortality were studied. The follow-up was unfortunately not available apart from the interval between surgery and discharge.

\section{Results}

The male to female ratio of patients was 2.8:1.

The youngest patient was a 36-year-old male and the oldest one was a 67-year-old lady. The mean age was 54.6 years old. The age and sex distribution of these patients is displayed in Table 1. Most of the patients (96.3\%) were above 40 .

All patients had history of IHD and therefore; LVA was a complication of MI.

We could obtain ECG recordings from 19 patients only. These were studied carefully. The typical LV aneurysm morphology (ST elevation seen $>2$ weeks follow-
Table 1 . The age and sex distribution.

\begin{tabular}{cccccc}
\hline Age (year) & $31-40$ & $41-50$ & $51-60$ & $61-70$ & Total \\
\hline Males & 1 & 8 & 5 & 6 & 20 \\
Females & 0 & 1 & 4 & 2 & 7 \\
Total & 1 & 9 & 9 & 8 & 27 \\
\hline
\end{tabular}

ing acute MI, most commonly in the precordial leads, with a concave or convex morphology, associated with well-formed Q- or QS-waves and a relatively small amplitude T-waves) was observed in 8 patients (42.1\%).

During the period of this study 1136 patients with IHD had CABG operations in IBCCS. Thus the overall rate of LVA repair to CABG operations was $2.4 \%$. Regarding distribution of patients over years of the study, we found that $62 \%$ of them were seen in the last three years.

Echocardiography was done in 25 patients. The ejection fraction values are shown in Table 2 . The vast majority (84.2\%) had subnormal values. Other echocardiographic findings are shown in Table 3.

The sites of aneurysm are displayed in Table 4: apical and antero-apical locations were the commonest.

Most patients in this study had multi-vessel CAD. Review of the coronary angiograms revealed about 46 stenotic or occlusive lesions in 27 patients as shown in Table 5 . The most frequently diseased artery was the LAD.

The perfusion charts of 25 patients were reviewed. Almost one third of patients (8) required IABP support mostly at the end of operation.

The operative procedures done to the patients in this study are shown in Table 6 . Twenty four patients (88.9\%) had CABG. Four patients (14.8\%) with an associated VSD had closure of these defects.

The type of repair of LV aneurysms is shown in Table 7. Linear repair and Dor technique were almost used equally. The details of myocardial revascularization are shown in Table 8. Most of the patients had multivessel coronary artery disease and received complete revascularization.

Regarding the outcome of patients with an associated VSD; one patient has died making a mortality of $25 \%$.

In regard to the duration of postoperative hospitalization, most patients (19, 70.4\%) stayed for 1 - 2 weeks.

The complications are displayed by Table 9 . The commonest was bleeding.

Five out of twenty-seven patients who were managed surgically had died (18.5\%). This represents death during the first hospitalization only. The mortality after discharge might be higher as there was no recordable follow up.

\section{Discussion}

The 27 patients with LVA repair represented $2.4 \%$ of the total 1136 patients who underwent CABG operations 
Table 2. Ejection fractions.

\begin{tabular}{ccccccccccc}
\hline \multicolumn{9}{c}{ Subnormal } & \multicolumn{4}{c}{ Normal $^{*}$} \\
\hline EF \% & $21-30$ & $31-40$ & $41-50$ & $51-55$ & $56-60$ & $61-70$ & $71-80$ & Not mentioned & Total \\
Pts, n (\%) & $1(5.3)$ & $7(36.8)$ & $6(31.5)$ & $2(10.6)$ & $1(5.3)$ & $1(5.3)$ & $1(5.3)$ & $6(24)$ & $25(100)$ \\
\hline
\end{tabular}

*Normal EF is $55 \%$ - $70 \%$ with a mean of $58 \%$.

Table 3. Other echocardiographic findings.

\begin{tabular}{|c|c|c|c|}
\hline Finding & Pts, n (\%) & Finding & Pts, n (\%) \\
\hline Aneurysm & $12(48)$ & MR & $8(32)$ \\
\hline Hypokinesia & $12(48)$ & $\mathrm{TR}$ & $3(12)$ \\
\hline Dyskinesia & $3(12)$ & & \\
\hline Akinesia & $5(20)$ & Dilatation of LV & $13(52)$ \\
\hline $\mathrm{P}^{*}$. effusion & $2(8)$ & Dilatation of RA & $2(6)$ \\
\hline Thrombus & $5(20)$ & Dilatation of RV & $3(12)$ \\
\hline
\end{tabular}

*P: pericardial; TR: tricuspid regurgitation; MR: mitral regurgitation; VSD: ventricular septal defect.

Table 4. Sites of aneurysms.

\begin{tabular}{ccccccc}
\hline Site & Anteroapical & Anteroseptal & Apical & Posteroinferior & Inferior & Total \\
\hline Pts, $\mathrm{n}(\%)$ & $9(33.3)$ & $2(7.4)$ & $10(37.1)$ & $2(7.4)$ & $4(14.8)$ & $27(100)$ \\
\hline
\end{tabular}

Table 5. Diseased vessels, n (\%).

\begin{tabular}{ccccc}
\hline RCA & LMS & LAD & CX & TOTAL \\
\hline $13(28.3)$ & $2(4.4)$ & $21(45.6)$ & $10(21.7)$ & $46(100)$
\end{tabular}

RCA: right coronary artery; LMS: left main stem; LAD: left anterior descending; CX: circumflex.

Table 6. Operative procedures.

\begin{tabular}{ccccc}
\hline Operation & LVA repair + CABG + VSD closure & LVA repair + CABG & LVA repair/no CABG & Total \\
\hline Pts, $\mathrm{n}(\%)$ & $4(14.8)$ & $20(74)$ & $3(11.2)$ & $27(100)$ \\
\hline
\end{tabular}

CABG: coronary artery bypass grafting.

Table 7. Types of repair.

\begin{tabular}{ccccccc}
\hline Type & Linear & Dor technique & Not specified & Patch & Linear + patch & Total \\
\hline Pts, n (\%) & $11(40.7)$ & $10(37.1)$ & $4(14.8)$ & $1(3.7)$ & $1(3.7)$ & $27(100)$ \\
\hline
\end{tabular}

Table 8. Details of myocardial revascularization.

\begin{tabular}{cccccc}
\hline No. of grafts & CABG X 1 & CABG X 2 & CABG X 3 & CABG X 4 & Total \\
\hline Pts, $\mathrm{n}(\%)$ & $1(4.2)$ & $7(29.2)$ & $8(33.3)$ & $8(33.3)$ & $24(100)$ \\
\hline
\end{tabular}

Table 9. Postoperative complications.

\begin{tabular}{cccccccc}
\hline Complication & Bleeding & Wound infection & Low CO & Uremia & Pleural effusion & Bed sore & Total \\
\hline Pts, $\mathbf{n}(\%)$ & $5(38.4)$ & $2(15.4)$ & $2(15.4)$ & $2(15.4)$ & $1(7.7)$ & $1(7.7)$ & $13(100)$ \\
\hline
\end{tabular}


during the period of the study in IBCCS. This figure is close to that reported by Antunes et al. from Portugal (2\%) [4].

\subsection{Age \& Sex Distribution}

LVA following MI has a similar age and sex distribution to IHD [4-10].

\subsection{Symptoms}

With regard to presenting symptoms, absence of angina pectoris and dyspnea as the predominating symptom was associated with early mortality according to a study by Vural et al. [11]. Moreover, lack of angina was an independent predictor for operative mortality [11]. It can be speculated that, preoperative angina, probably but not necessarily, may indicate viable myocardial tissue existence, which is capable of generating power during systole, more compliant during diastole and has less compromising effect on ventricular geometry when compared to a totally fibrotic aneurysmal sac [11].

\subsection{Electrocardiography}

The typical LV aneurysm morphology in the ECG described earlier in the Results was observed in 8 patients (42.1\%) only. This ECG pattern has a sensitivity of 38\% and a specificity of $84 \%$ for the diagnosis of ventricular aneurysm [7].

\subsection{Incidence}

$62 \%$ of patients were seen in the last three years of the study. This could be related to a real increase in the incidence of IHD and its complication like LVA, a concomitant rise in CABG operations, a better diagnosis of LVA, increased awareness of cardiologists about the role of surgery in LVA and thus more referral of cases and a significant increase in the number of efficient young coronary surgeons capable of performing LVA repair with concomitant CABG.

\subsection{Diagnosis}

Echocardiography has a sensitivity and specificity of 93\% and 94\%, respectively, for detecting LV aneurysm, representing the most frequent and easily applied test for such an anatomic abnormality. Left ventriculography, however, remains the gold standard for the diagnosis [9].

\subsection{Ejection Fraction}

The vast majority of our patients (84.2\%) had subnormal EF values. Results of many studies have shown that the ejection fraction of the total left ventricle is an important predictor of the outcome of open heart surgery $[11,12]$.
Ventricular ejection fraction improves following aneurysm repair whether linear or patch technique is used [1].

\subsection{Other Echocardiographic Findings}

In the present study, echocardiography was very useful. This is evident by looking at Table 4 which displayed many studied parameters. Mural thrombi were found in $20 \%$ of patients exactly the same as reported by Mangschau et al. [13] and were removed surgically. Four postischemic VSDs were accurately diagnosed and fixed surgically thereafter.

\subsection{Location}

In this study, anterior (11; 40.7\%) and apical aneurysms (10; 37.1\%) were the commonest. In clinical reports, LVA is usually located in the anterior wall, whereas infero-posterior or postero-lateral aneurysms are less common. Postinfarction LVA follows pathology of the LADdiagonal system (anterior aneurysm), circumflex branches (posterolateral aneurysm), or right coronary artery (inferoposterior aneurysm) [14]. In our series, a significant LAD lesion was present in 21 patients (77.8\%). The prevalence of inferoposterior aneurysms is significantly higher in autopsy series than in clinical reports [14]. This may be due to the extensive infarction necessary for LVA formation. When this occurs in the inferoposterior wall, the result is often acute, severe mitral regurgitation and the patient dies in the acute phase rather than develops LVA [14].

\subsection{Linear vs. Patch Repair}

In the present series, linear and Dor repair were almost equally used (11 patients, $40.7 \%$ in each group), whereas the method of repair was not clear in 4 patients (14.8\%). The basis on which patients were offered either type of repair is not known. Generally, the choice of repair technique should not be made randomly, but depending on factors such as size and extension of scar tissue [11].

Although aneurysmectomy has been performed for almost five decades, the most appropriate surgical approach to a patient with a dyskinetic LVA is still controversial [4]. Antunes et al. believed that the technique of repair of postinfarction dyskinetic LVAs should be adapted in each patient to the cavity size and shape, and the dimension of the scar [4]. Unduly wide excision of the scar area and linear closure of the LV defect might lead to deformation of the $\mathrm{LV}$ chamber and a reduction in LV diastolic volume [15].

\subsection{Impact of Coronary Revascularization}

CABG is one of the important components of LVA surgery, and the revascularization rate varies in the literature 
from $68 \%$ to $100 \%$ [3] Twenty four patients in this study (88.9\%) had CABG; which goes with the international standard. Most of the patients had multi-vessel coronary artery disease and received complete revascularization as shown in Table 9. Although the surgical risk is increased, patients with low LVEF and multi-vessel disease have a particular survival benefit after CABG [14]. The biological basis for this is recruitment of hibernating myocardium [14].

\subsection{When CABG Is Not Added!}

Three patients in this study had LVA repair without CABG. Two patients survived while the third (having total occlusion of RCA) died. It is noteworthy that RCA disease is associated with low cardiac output (CO) on multivariate analysis [3] which probably was the cause of death in this patient. In a study on 303 patients with LVA from Sweden, Stahle et al. reported an early mortality of $23 \%$ in patients who underwent aneurysm resection alone and $8.1 \%$ in cases of aneurysm resection with CABG [6]. This emphasizes the importance of concomitant CABG with LVA repair. Vural et al. in a retrospective analysis of 248 patients also found that concomitant CABG reduced the incidence of low CO state [11].

\subsection{LVA + VSD}

This is an important complication of MI that has been commonly associated with progression to death [16]. Surgery is routinely performed in patients with acute VSD during MI [16]. Schlichter et al. reported a 3\% incidence of VSD in a series of 102 patients with LVA [17]. Acquired VSD was first surgically repaired by Cooley et al. (1957) using CPB and hypothermia [17]. In 1962, Collis et al. described the repair of a VSD and LVA in a man of 59 [17]. When LVA coexists with a VSD, there is an obvious route for access to the septum since the left ventricular myocardium is already damaged [17]. The approach from the left which is thus afforded is ideal, first in that the septum on this side is smoother and the defect thus is more easily defined, and secondly the left ventricular pressure keeps the patch in contact with the septum, whereas a patch applied from the right side may be forced away from the septum [17]. Lazopoulos et al. described a case of giant LVA and VSD following a silent MI managed by an endoventricular circular plasty (Dor procedure), interrupted suturing of ventricular septum and CABG [18].

In the present series, 4 patients had LVA + VASD (14.8\%). One patient died (25\% mortality). This is higher than what was reported by Olearchyk et al. (20\%) [19].

\subsection{Morbidity}

The commonest postoperative complication was bleeding.
In other studies $[3,4]$ low CO was on the top of the list.

\subsection{Overall Mortality}

5 out of 27 patients who were managed surgically had died (18.5\%). In a collection of 3439 operations for LVA performed between 1972 and 1987, hospital mortality was $9.9 \%$ and ranged from $2 \%$ to $19 \%$ [1] and it has recently fallen to $3 \%$ to $7 \%$ in the last decade [1]. In view of these figures, our mortality is obviously high. The most likely reason is the small number of patients and the limited experience in the management of this condition. In regard to the possible causes of death, postoperative bleeding was blamed in 2, low $\mathrm{CO}$ in 1 while it remained unknown in 2 patients. Low CO accounted for 34\% of early mortality in Vural et al. series [11]. Mukkadirov et $a l$. also believes that severe low CO is one of the main causes of early mortality after aneurysmectomy [3].

\subsection{Perfusion Time and IABP}

The mean perfusion time in this study was 100 minutes, higher than that reported by Antunes et al. (82.7 minutes) [4]. Long periods of aortic-cross clamping have detrimental effects during weaning from CPB [20].

IABP was used in 8 patients (32\%) in this study, whereas Eid used it in $26.7 \%$ of his patients [20]. IABP has become a prerequisite for surgical repair of LVA [20] especially in patients with compromised LV function [20].

\section{REFERENCES}

[1] G. D. Di and L. J Ei, “Left Ventricular Aneurysm,” In: L. H. Cohn and L. H. Edmunds Jr., Eds., Cardiac Surgery in the Adult, McGraw-Hill, New York, 2003, pp. 771-788.

[2] M. Mukaddirov, R. G. Demaria, L. P. Perrault, J.-M. Fradier and B. Albat, "Reconstructive Surgery of Post Infarction Left Ventricular Aneurysms: Techniques and Unsolved Problems,” European Journal of Cardio-Thoracic Surgery, Vol. 34, No. 2, 2008, pp. 256-261. http://dx.doi.org/10.1016/j.ejcts.2008.03.061

[3] M. Mukkadirov, J.-M. Frapier, R. G. Demaria and B. Albat, "Surgical Treatment of Post Infarction Left Ventricular Aneurysms: Linear vs. Patch Plasty Repair," Interactive Cardiovascular and Thoracic Surgery, Vol. 7, No. 2, 2008, pp. 256-261.

http://dx.doi.org/10.1510/icvts.2007.160093

[4] P. E. Antunes, R. Silva, J. F. de Oliveira and M. J. Antunes, "Left Ventricular Aneurysms: Early and Late LongTerm Results of Two Types of Repair,” European Journal of Cardio-Thoracic Surgery, Vol. 27, No. 2, 2005, pp. 210-215. http://dx.doi.org/10.1016/j.ejcts.2004.11.010

[5] L. Menicanti and M. Di Donato, "Left Ventricular Aneurysm/Reshaping Techniques,” MMCTS, Vol. 2005, No. 0425, 2005.

http://dx.doi.org/10.1510/mmcts.2004.000596 
[6] E. Stahle, R. Bergestrom, S. O. Nystrom, B. Edlund, I. Sjorgren and L. Holmberg, "Surgical Treatment of Left Ventricular Aneurysm-Assessment of Risk Factors for Early and Late Mortality," European Journal of CardioThoracic Surgery, Vol. 8, No. 2, 1994, pp. 67-73. http://dx.doi.org/10.1016/1010-7940(94)90094-9

[7] Ed Burns, ECG Library, Life in the fastlane.com.

[8] J. Edhouse, et al., "ABC of Clinical Electrocardiography," BMJ, Vol. 324, No. 20, 2002, pp. 963-969. www.bmj.com

[9] W. J. Brady, R. A. Harrigan and T. Chan, "Diagnosis: Left Ventricular Aneurysm,” Cases in Electrocardiography. Emergency Medicine News, Vol. 28, No. 6, 2006, p 38. http://dx.doi.org/10.1097/00132981-200606000-00025

[10] V. N. Singh and J. A. Strom, "Ventricular Aneurysm Imaging,” 2012.

[11] K. M. Vural, E. Sener, M. A. Ozatic, O. Tasdemir and K. Bayazit, "Left Ventricular Aneurysm Repair: An Assessment of Surgical Treatment Modalities," European Journal of Cardio-Thoracic Surgery, Vol. 13, No. 1, 1998, pp. 49-56.

http://dx.doi.org/10.1016/S1010-7940(97)00287-X

[12] L. E. Watson, D. W. Dickhaus and R. H. Martin, "Left Ventricular Aneurysm: Preoperative Haemodynamics, Chamber Volume and Results of Aneurysmectomy," Circulation, Vol. 52, No. 5, 1975, pp. 868-873. http://dx.doi.org/10.1161/01.CIR.52.5.868

[13] A. Mangschau, S. Simonsen, M. Abdelnoor, B. Laake and O. Geiran, "Evaluation of Left Ventricular Aneurysm Resection: A Prospective Study of Clinical and Haemodynamic Characteristics," European Journal of CardioThoracic Surgery, Vol. 3, No. 1, 1989, pp. 58-64. http://dx.doi.org/10.1016/1010-7940(89)90013-4
[14] R. Lundblad, M. Abdelnoor and J. L. Svennevig, "Repair of Left Ventricular Aneurysm: Surgical Risk and LongTerm Survival,” The Annals of Thoracic Surgery, Vol. 76, No. 3, 2003, pp. 719-725. http://dx.doi.org/10.1016/S0003-4975(03)00677-5

[15] A. V. Marchenko, A. M. Cherniavsky, T. L. Volokitina, A. S. Alsov and A. M. Karaskov, "Left Ventricular Dimension and Shape after Postinfarction Aneurysm Repair," European Journal of Cardio-Thoracic Surgery, Vol. 27, No. 3, 2005, pp. 475-480. http://dx.doi.org/10.1016/j.ejcts.2004.12.025

[16] O. Shindler, A. Spotnitz and D. Shindler, "Aneurysm and Ventricular Septal Defect Following Myocardial Infarction,” E-Chocardiography Journal, 1995. http://rwjms1.umdnj.edu/Shindler/vsdaneurysm.html

[17] A. D. Heath, A. M. Harris and M. P. Wright, "Clinical Features and Repair of Ventricular Septal Defect and Left Ventricular Aneurysm Complicating Myocardial Infarction,” British Heart Journal, Vol. 32, No. 6, 1970, pp. 863-866. http://dx.doi.org/10.1136/hrt.32.6.863

[18] G. Lazopoulos, M. Manns-Kantartzis and M. Kantartzis, "Giant Left Ventricular Aneurysm and Intra-Ventricular Septal Defect after Silent Myocardial Infarction," The Hellenic Journal of Cardiology, Vol. 50, 2009, pp. 142143.

[19] A. S. Olearchyk, G. M. Lemole and P. M. Spagna, "Left Ventricular Aneurysm. Ten Years Experience in Surgical Treatment of 244 Cases. Improved Clinical Status, Hemodynamics and Long-Term Longevity," The Journal of Thoracic and Cardiovascular Surgery, Vol. 88, No. 4, 1984, pp. 544-553.

[20] H. E. Eid, "Role of Intra-Aortic Balloon Pump in Left Ventricular Endoaneurysmorrhaphy," Asian Cardiovascular and Thoracic Annals, Vol. 7, No. 4, 1999, pp. 276281. http://dx.doi.org/10.1177/021849239900700406 